\title{
Editorial
}

\section{Omicron; past, current and future perspectives}

\author{
Pottathil Shinu', Rajesh Bareja ${ }^{2, *}$ \\ ${ }^{1}$ Dept. of Biomedical Sciences, College of Clinical Pharmacy, King Faisal University, Al Ahsa, Saudi Arabia \\ ${ }^{2}$ Dept. of Microbiology, World college of Medical Sciences \& Research, Jhajjar, Haryana, India
}

\section{A R T I C L E I N F O}

Article history:

Received 20-01-2022

Accepted 27-01-2022

Available online 12-02-2022

\begin{abstract}
This is an Open Access (OA) journal, and articles are distributed under the terms of the Creative Commons Attribution-NonCommercial-ShareAlike 4.0 License, which allows others to remix, tweak, and build upon the work non-commercially, as long as appropriate credit is given and the new creations are licensed under the identical terms.

For reprints contact: reprint@ipinnovative.com
\end{abstract}

The World Health Organization (WHO) declared newly mutated severe acute respiratory syndrome coronavirus 2 (SARS-CoV-2) variant Omicron (B.1.1.529) as a new variant of concern (VOC) on November 26, 2021. This variant possess multiple mutations on the spike (S) proteins, the main protein of SARS-CoV-2 that act as antigenic target for production of antibodies against immunization or infections. However, the most prevalent Delta variant which carried $5 \mathrm{~S}$ protein mutations only. Considering these fact, a "panic button" has been initiated across the world including travel restrictions in order to limit the rapid spread of the Omicron variant. ${ }^{1,2}$

Various factors that influenced the emergence of Omicron variants may include the presence of unknown animal reservoirs, low immunization rate among Africans (as the first case was reported in Botswana in November), high spike protein mutation rates (32 mutations) on the SARS-CoV-2, which would have indirectly increased the ability of the virus to attach with ACE2 receptor on human cells. ${ }^{3}$ On December 13, 2021, first case of death was confirmed due to Omicron variant of SARS-CoV2 in the UK. ${ }^{1}$ Mutational data of SARS-CoV-2 showed some concerning mutations (N501Y, D614G, K417N, and $\mathrm{T} 478 \mathrm{~K}$ ) along with new mutations present in the Omicron variant which enhanced the chances of reinfection and partial resistance to current vaccines. ${ }^{4}$ However, there are

\footnotetext{
* Corresponding author.

E-mail address: rajeshbareja@gmail.com (R. Bareja).
}

no adequate data available to analyze rate of transmission of newly mutated Omicron variant. Data available from South Africa suggest that person-to-person transmission is much faster by Omicron variant strains. ${ }^{5}$ Furthermore, the reports shows that Omicron variant can cause reinfection in previously SARS-CoV-2 infected patients. ${ }^{6}$ It is still being analyzed the efficacy of vaccine against new variant of SARS-CoV-2. Two doses of vaccination could provide more than $90 \%$ protection against severe disease when patients were infected with the Delta variant. ${ }^{7}$ However, it was reported that individuals who received mRNA vaccine shots showed robust neutralization of the Omicron variant indicating presence of significant cross-reactivities neutralizing antibodies. Presence of neutralizing antibodies in these patients suggest that current COVID-19 mRNA vaccines may have a protective effect in patients infected with Omicron variant. This is most likely that most of the epitopes on spikes proteins of the Omicron variant are not mutated against the vaccine-induced $T$ cells, which is formed in the vaccinated individuals. ${ }^{7}$

This situation alarmed the health agencies across the world to strengthen surveillance, awareness among public about vaccination and follow public health measures while avoiding overreaction ${ }^{4}$. Various researchers reported that infection with Omicron variant shows $15 \%-80 \%$ reduced risk of hospitalization as compared to infection with delta variant. It is also evident from various surveillance data that the latest omicron-wave having significantly fewer 
hospital admissions and mortality rates than the delta variant pandemic. $^{8}$ However, emergence of Omicron, a New SARS-CoV-2 variant, reiterate that the epidemic is not going to end soon. This situation demands the importance of vaccination, awareness among public to continue preexisting guidelines like social distancing, wearing masks, handwashing at regular intervals to restrict the spread of ongoing pandemic.

Mutation is a continuous heritable process resulting in formation of multiple genetic variants. It is expected that waves of heavy infection with the new variant of Omicron may continue. However, the severity of infection with Omicron is still unknown. Therefore, it is highly recommended to perform genome sequencing of all the SARS COV-2 cases, maintenance of social distancing, vaccination drives and isolation of SARS COV-2 patients that might help in improving the current situation.

\section{Conflict of Interest}

The authors declare that there is no conflict of interest.

\section{Source of Funding}

None.

\section{References}

1. World Health Organization Classification of Omicron (B.1.1.529): SARS-CoV-2 Variant of Concern. Available from: https: //www.who.int/news/item/26-11-2021-classification-of-omicron(b.1.1.529)-sars-cov-2-variant-of-concern.

2. Gao SJ, Guo H, Luo G. Omicron variant (B.1.1.529) of SARS-CoV-2, a global urgent public health alert! J Med Virol. 2021 ;o1:10.1002/jmv.27563.

3. Kupferschmidt K. Where did 'weird' Omicron come from? Science. 2021;374(6572):1179. d01:10.126/scienceacxy/38

4. Araf Y, Akter F, Tang YD, Fatemi R, Parvez M, Zheng C, et al. Omicron variant of SARS-CoV-2: Genomics, transmissibility, and responses to current COVID-19 vaccines. J Med Virol. 2022;do1:10.1002/jmv.27588.

5. Foxnews. Omicron is better at evading vaccines, new COVID-19 transmissibility data confirms. Available from: https://www.foxnews. com/world/omicron-vaccines-new-covid-transmissibility-data.

6. Pulliam JR, Van Schalkwyk C, Govender N, Gottberg AV, Cohen C, Groome $\mathrm{MJ}$, et al. Increased risk of SARS-CoV-2 reinfection associated with emergence of the Omicron variant in South Africa. MedRxiv.



7. Hoffmann M, Krüger N, Schulz S, Cossmann A, Rocha C, Kempf A, et al. The Omicron variant is highly resistant against antibody-mediated neutralization-implications for control of the COVID-19 pandemic. Cell. 2021;185(3):447-56. đ01:10.1016/].cell.2021.12.032

8. Christie B. Covid-19: Early studies give hope omicron is milder than other variants. BMJ. 2021;375:3144. do1:10.1136/bm].n3144.

\section{Author biography}

Pottathil Shinu, Assistant Professor

Rajesh Bareja, Professor

Cite this article: Shinu P, Bareja R. Omicron; past, current and future perspectives. IP Int J Med Microbiol Trop Dis 2022;8(1):1-2. 